\title{
Videolaringoscopía para intubación pediátrica, en comparación con la intubación orotraqueal clásica.
}

Video laryngoscopy for pediatric intubation, compared to classic orotracheal intubation.

\section{Juan Patricio Palacios Alvear ${ }^{1}$ Juan Pablo Pacheco Bacuilima ${ }^{1}$}

1 Departamento de Postgrado de Anestesiología, Facultad de Ciencias Médicas, Universidad de Cuenca-Ecuador.

Recibido: 2 Marzo 2020

Aceptado: 12 Julio 2020

Publicado: 31Agosto 2020

Membrete bibliográfico:

Palacios J, Pacheco J. Videolaringoscopía para intubación pediátrica, en comparación con la intubación orotraqueal clásica. Ecuador 2018. Rev. Ecuat. Pediatría 2020:21(2): Artículo 13:1 9.

Copyright Palacios J, et al. Este artículo es distribuido bajo los términos de Creative Commons Attribution License CC BY-NC-SA 4.0, el cual permite el uso y redistribución citando la fuente $y$ al autor original sin fines comerciales.

\section{STROBE Statement}

\section{RESUMEN}

Introducción: Un aspecto relevante en anestesia pediátrica es el manejo de la vía aérea. Diferencias anatómicas de esta población, hace que sean más susceptibles a dispositivos diseñados para su manejo. La videolaringoscopía mejora la visión panorámica de la glotis. El objetivo del presente estudio fue comparar el uso de la videolaringoscopía con la laringoscopía directa para intubación orotraqueal, en pacientes pediátricos.

Métodos: Con un diseño descriptivo observacional, se estudiaron 276 casos, pacientes de 5 a $\mathbb{7}$ años, sometidos a cirugía planificada, con anestesia general más intubación orotraqueal. El especialista decidió el dispositivo para manejo. Los datos se transcribieron de los formularios y analizados con el programa SPSS 22.0. El análisis descriptivo se basó en mediana, moda, promedio, valor mínimo, valor máximo. Medidas de dispersión desvío estándar y rango. Se consideró un valor de $p \leq 0.05$ como estadísticamente significativo.

Resultados: La edad promedio del estudio fue 9.83 años. Con laringoscopía directa $97.4 \%$ se realizó la intubación al primer intento y con videolaringoscopía el $88,4 \%(P=0.003)$. En el $94.2 \%$ de casos de la ringoscopía directa se realizó una intubación exitosa en menos de 10 segundos, con videolaringoscopía fue del $75.2 \%(P<0.001)$. Complicaciones se presentaron en el $6.6 \%$ de intubaciones con videolaringoscopía versus el $2.6 \%$ con la ringoscopía convencional ( $P=0.103$ ). El 56.2\% de especialistas prefieren laringoscopía directa para manejar una vía aérea pediátrica sin predictores de dificultad.

Conclusiones: La videolaringoscopía brinda un apoyo adicional en el manejo de la vía aérea de rutina, amerita siempre conocimientos y destrezas previas. La evidencia para avalar una técnica por sobre otra es insuficiente.

\section{Palabras Claves:}

DeCS: Vía aérea, Videolaringoscopía, Tiempo de intubación, Complicaciones, Número de intentos.

\footnotetext{
* Autor para correspondencia.
}

Correo electrónico: juanpacho8903@gmail.com (Juan Patricio Palacios Alvear) Cl: 0103896908 Hospital Vicente Corral Moscoso, Anestesiología. Código Postal 010107 
Received: March 2, 2020

Accepted: July D, 2020

Published: August 31, 2020

Bibliographic letterhead:

Palacios J. Video laryngoscopy for pediatric intubation, compared to classic orotracheal intubation. Rev. Ecuat. Pediatría 2020; Article 13:1 9 .

Copyright Palacios ], et al. This article is distributed under the terms of the Creative Commons Attribution License CC BY-NC-SA 4.0, which allows the use and redistribution citing the source and the original author without commercial purposes.

STROBE Statement

\section{INTRODUCCIÓN}

Una parte fundamental en la práctica de la anestesia pediátrica es el abordaje de la vía aérea (VA). Las características anatómicas de esta población, y las diferencias estructurales en comparación con los adultos, hacen que sean más susceptibles a complicaciones causadas por los dispositivos de video diseñados para su abordaje. Estudios de imágenes de VA en niños, identifican la glotis como la parte más angosta, y la laringe más cilíndrica que cónica. Estos hallazgos generan discrepancias al momento de escoger un dispositivo de manejo ${ }^{1}$.
Si bien, no son frecuentes las complicaciones relacionadas con el manejo de la VA pediátrica, en ocasiones generan morbilidad en la población vulnerable como recién nacidos y lactantes menores. Adversidades como una ventilación inadecuada, obstrucción bronquial, apnea o intubación difícil, son causas relacionadas con morbimortalidad en esta población. Conocer las ventajas y limitaciones de las diferentes técnicas de intubación orotraqueal pediátrica es un punto de partida para optimizar su abordaje. La videolaringoscopía (VL), se está 
convirtiendo en un apoyo fundamental para la intubación orotraqueal pediátrica².

El ASA Closed Claims, revela que el $34 \%$ de las demandas a anestesiólogos tienen relación con eventos en la vía aérea, y representan el $10 \%$ de las denuncias presentadas contra los profesionales de la salud en el ámbito pediátrico ${ }^{3}$.

Esta investigación beneficia a médicos tratantes y encargados del manejo de la VA pediátrica, estableciendo protocolos de manejo adecuado, capacidad de anticiparse a una posible vía aérea difícil (VAD), o contar con una herramienta de apoyo adicional en el caso de una vía aérea difícil no prevista. De manera directa a los pacientes que recibirán un trato individualizado en cuanto a valoración y manejo de vía aérea. El objetivo del presente estudio fue comparar el uso de la videolaringoscopía con la laringoscopía directa para intubación orotraqueal, en pacientes pediátricos.

\section{POBLACIÓN Y MÉTODOS}

\section{Diseño del estudio}

Se realizó un estudio descriptivo, observacional, analítico.

\section{Escenario}

Los quirófanos del Hospital Vicente Corral Moscoso, perteneciente al Ministerio de Salud Pública, CuencaEcuador. Zona de salud \#6, Av. Los Arupos y Av. 12 de abril, en el periodo 1de enero - a 31de diciembre del 2018. El período de campo fue considerado como período de reclutamiento y exposición. El seguimiento de los resultados se terminó el 7 de Marzo del 2019 y el período de recopilación de datos terminó el 7 de Abril del 2019.

\section{Participantes}

Se tomó como universo de estudio, a los pacientes de 5 a 17 años de edad, 980 en total, que acudieron al Hospital Vicente Corral Moscoso en el periodo enero diciembre 2017, provenientes de las áreas de hospitalización y consulta externa; quienes fueron sometidos a una cirugía planificada mediante anestesia general más intubación orotraqueal.

\section{Variables}

Edad, Sexo, Estado Nutricional, Preferencia de laringoscopia del operador, Tiempo de intubación, Número de intentos hasta realizar una intubación, Complicaciones (sangrado, edema, trauma dental, dificultad en la técnica, otros).

\section{Fuentes de datos}

La fuente fue directa, posterior a la intubación exitosa, se procedió a la recolección de datos para la elaboración de las tablas estadísticas posteriores. Se utilizó un formulario, en el cual se transcribieron los datos del paciente, tiempo en segundos de intubación, número de intentos, preferencia del operador y complicaciones presentadas, en los pacientes 5 a 17 años, sometidos a cirugía planificada más intubación orotraqueal en la institución de estudio. Se tomó como división natural la preferencia de intubación de los anestesiólogos, conformándose el grupo control con pacientes intubados con laringoscopia y comparados con pacientes intubados con videolaringoscopía.

\section{Procedimientos para la recolección de información} e instrumentos.

El tiempo de intubación se calculó en segundos, se inició el cronometraje cuando el médico a cargo de la VA, culminó los 3 minutos de ventilación asistida y se dispuso a la intubación, se usó un cronometro digital para obtener el tiempo real en segundos.

El número de intentos valoró, cuantas veces el médico anestesiólogo o residente bajo supervisión del mismo, necesitó retirar el tubo para redirigir el mismo y obtener una intubación adecuada. Teniendo presente el sesgo que se presentó al momento de la intubación, debido a las diferencias de nivel en la curva de aprendizaje entre un anestesiólogo y un médico especialista en formación.

Las complicaciones se las observó en el momento de la laringoscopia sea directa o indirecta, dentro de las cuales tuvimos trauma dental, sangrado, dificultad en la técnica, edema. 
La edad en años se calculó de acuerdo con los datos tomados de cédula de ciudadanía, o a su vez partida de nacimiento, debidamente registrados en la historia clínica.

El peso fue calculado mediante el uso de una balanza mecánica, marca PERFECTCH con capacidad para $400 \mathrm{~kg}$. Se colocó a los niños directamente en la balanza, con la menor cantidad de ropa posible, para obtener el peso real, siempre se contó con el apoyo del representante, los valores fueron registrados en $\mathrm{kg}$.

La talla se calculó mediante un tallímetro mecánico, el mismo que se encontraba acoplado a la balanza mecánica marca PERFECTCH antes descrita, con una medición de altura de 50 a 200 cms., el cual proporcionó el dato exacto en cms.

El estado nutricional se calculó de acuerdo a los percentiles manejados por la Organización Mundial de la Salud.

El tipo de instrumento usado para la intubación lo decidió el especialista a cargo de la intubación según su familiaridad con los dispositivos y destrezas.

\section{Control de las fuentes de sesgo.}

Se excluyeron historias clínicas cuyos datos no estuvieron completos, se evitó la imputación de datos perdidos o excluidos. El protocolo de este estudio fue pre aprobado por el Comité de docencia Institucional y el Comité de Bioética de la Universidad de Cuenca. No se constituye un estudio experimental ya que se observó el procedimiento normal del departamento de anestesia y las preferencias de intubación de los médicos especialistas.

\section{Tamaño del estudio}

La muestra fue probabilística, con un Universo de 980 pacientes, un nivel de confianza 95\% 196, Margen de error: $5 \%$ 0.05, probabilidad de ocurrencia del evento: 0.5, probabilidad de no ocurrencia: 0.5 . Se usó la fórmula de cálculo de una muestra conocida para proporción en EPI-Info 7.0. El tamaño calculado para la muestra fue de 276 pacientes.

\section{Manejo de variables cuantitativas}

Las variables discretas se presentan en frecuencia y sus porcentajes (\%). Se realizaron tablas estadísticas de acuerdo a cada variable. En el análisis de los datos estadísticos descriptivos, se utilizó: mediana, moda, promedio, valor mínimo, valor máximo, y medidas de dispersión como rango y desvió estándar. Se consideró un valor de $P \leq 0.05$ como estadisticamente significativo.

\section{Métodos Estadísticos}

Los datos que fueron recolectados en el formulario, se ingresaron en el programa estadístico SPSS de IBM 22. 0 versión libre.

\section{RESULTADOS}

\section{Caracterización sociodemográfica de la población en estudio.}

El promedio de edad del grupo de estudio es de 9.83 años, la mediana 9 años, la moda 5 años, el rango es de 12 años, valor mínimo 5 años y valor máximo 17 años. El grupo de edad más frecuente es de 5 a 10 años con el $612 \%$

Hubo un predominio del sexo masculino $58.3 \% \mathrm{El}$ estado nutricional normal es más frecuente con el $56.9 \%$ Laringoscopia directa con el $56.2 \%$ es el método más frecuente para intubación orotraqueal, por sobre el 43.8\%de videolaringoscopía (Tabla 1).

Tabla 1 Descripción de la población estudiada

\begin{tabular}{|c|c|c|c|}
\hline & & $\mathrm{n}$ & $\%$ \\
\hline \multirow{2}{*}{ Edad } & 5- 10 años & 169 & $612 \%$ \\
\hline & ㄲ- 17 años & 107 & $38.8 \%$ \\
\hline \multirow{2}{*}{ Sexo } & Hombre & 161 & $58.3 \%$ \\
\hline & Mujer & 115 & $417 \%$ \\
\hline \multirow{4}{*}{$\begin{array}{l}\text { Índice } \\
\text { masa } \\
\text { corporal }\end{array}$} & Peso bajo & 35 & $12.7 \%$ \\
\hline & Normal & 157 & $56.9 \%$ \\
\hline & Sobrepeso & 35 & $12.7 \%$ \\
\hline & Obesidad & 49 & $17.8 \%$ \\
\hline \multirow{2}{*}{$\begin{array}{l}\text { Preferencia } \\
\text { de intubación }\end{array}$} & Laringoscopía & 155 & $56.2 \%$ \\
\hline & Video-laringoscopía & 21 & $43.8 \%$ \\
\hline $\begin{array}{lr}\text { por } & \text { el } \\
\text { anestesiólogo }\end{array}$ & & & \\
\hline
\end{tabular}


Instrumento de intubación y su relación con número de intentos.

Con la laringoscopia directa el $\mathbf{9 7 . 4 \%}$ se realizó la intubación al primer intento. Con videolaringoscopía el $88.4 \%$ Con videolaringoscopía el $116 \%$ de intubaciones necesito más de un intento, el $2.6 \%$ con laringoscopia. La diferencia es significativa $(P=0.003)$ (Tabla 2).

Tabla 2. Comparaciones entre técnicas

\begin{tabular}{lccc}
\hline & $\begin{array}{c}\mathrm{LD} \\
\mathrm{n}=15 \%\end{array}$ & $\begin{array}{c}\mathrm{V} \\
\mathrm{n}=\mathrm{D} 1\end{array}$ & $P$ \\
\hline Éxito al ler & $151(97.4 \%$ & $107(88.4 \%)$ & 0.003 \\
intento & & & \\
\cline { 2 - 4 } Complicaciones & $4(2.6 \%$ & $8(6.6 \%)$ & 0.103 \\
Trauma Dental & $3(19 \%)$ & $5(4.1 \%)$ & 0.103 \\
Sangrado & $1(0.6 \%)$ & $0(0 \%)$ & 0.103 \\
\cline { 2 - 4 } $\begin{array}{l}\text { Dificultad en la } \\
\text { técnica }\end{array}$ & $3(19 \%)$ & $21(17.4 \%)$ & 0.01 \\
Tiempo de & & & \\
intubación de 5 & $(94.2 \%)$ & & \\
a 10" vs & & & \\
11a 30" & $8(5.2 \%$ & $30(24.8 \%)$ & \\
\hline
\end{tabular}

LD: Laringoscopía directa. VL: video laringoscopía.

\section{Instrumento de intubación y su relación con las complicaciones.}

En la videolaringoscopía el 93.4\% de intubaciones se realizó sin complicaciones, mientras que con laringoscopía directa se realizó el $97.4 \%(P=0.103)$ no estadísticamente significativo. Mayor complejidad en la técnica de intubación mediante videolaringoscopía con un $17.4 \%$ versus $19 \%$ con laringoscopia directa (Tabla 2).

\section{Instrumento de intubación y su relación con tiempos de intubación.}

Con laringoscopia directa el $94.2 \%$ de los casos, se realizó una intubación entre 5 a 10 segundos, en relación al $75.2 \%$ con videolaringoscopía. El $24.8 \%$ del porcentaje de videolaringoscopía se realizó entre 11 a 30 segundos a comparación del 5.2\% con laringoscopía directa. Una intubación mayor a 30 segundos se presentó con laringoscopía convencional. Esto es estadísticamente significativo $(P \varangle 0.0001)$

\section{DISCUSIÓN}

Existen muy pocos estudios comparativos entre LD y VL en población pediátrica, la mayoría basa su estudio en adultos o maniquies. Con el fin de realizar un estudio comparativo y conocer el impacto de las técnicas de intubación pediátrica en nuestro medio, se desarrolló el tema Videolaringoscopía para intubación pediátrica, en comparación con la intubación orotraqueal clásica. Por un lado, el laringoscopio convencional siendo el más utilizado en las salas de quirófano por la simplicidad en su uso, costos y durabilidad, por otro lado, como método de VL el Vividtrac, ambos equipos con escasa evidencia en el uso rutinario de niños ${ }^{4-6}$. Una particularidad de nuestro estudio, fue que se realizó en población infantil sin predictores de VAD, la mayoría de estudios se basan en pacientes pediátricos con VAD reconocida, con malformaciones orofaríngeas o sindromes característicos que comprometen las vías respiratorias, lo que pueden denotar alteraciones en valores estadísticos resultantes, debido a que no se encuentran en un mismo rango de dificultad.

El promedio de edad del grupo de estudio fue de 9.83 años, la mediana 9 años, la moda 5 años, el rango es de 12 años, valor mínimo 5 años y valor máximo 17 años. El grupo de edad más frecuente fue 5 a 10 años con el $61.2 \%$, predominio del sexo masculino $58.3 \%$, el estado nutricional normal es más frecuente con el $56.9 \%$, seguido por los obesos con un $17.8 \%$.

Teniendo en cuenta la intubación en el primer intento, con LD se realizó el $97.4 \%$ de los casos, mientras que con VL las cifras descendieron a un $88.4 \%$. Lo que traduce que el $11.6 \%$ del total de intubaciones con VL se realizó en al menos 2 intentos, en comparación con LD que representó el $2.6 \%$ con $P=0.003$. En términos generales, estás diferencias debieran explicarse en gran parte a las características anatomo-fisiológicas de la población en estudio, el tamaño del dispositivo con relación al diámetro bucal y lengua prominente, que complican la maniobrabilidad del operador. Algunas estructuras anatómicas quedan fuera del campo de visión del video laringoscopio, las cuales dificultan el correcto deslizamiento del tubo endotraqueal desde la boca hacia la tráquea, 
obligando a redireccionar el mismo para una intubación satisfactoria. Algunos especialistas y médicos posgradistas participantes de este estudio, a pesar de tener experiencia en intubación con video laringoscopios en adultos, se les presento cierto grado de dificultad en la población pediátrica. Las tasas de éxito o fracaso no solamente se limitan a condiciones del anestesiólogo, dependen también de las características propias de cada paciente y del grupo etario $^{7-10}$.

Ramirez-Hernandez, y cols, en su estudio Videolaringoscopio airtraq versus fibroscopio bonfils para intubación orotraqueal electiva en población pediátrica, manifiesta que mediante videolaringoscopía un 33.3\% necesitó de más de un intento de intubación, en nuestro estudio se presentó un $11.6 \%{ }^{11}$. Lingappan y cols, en una revisión sistemática de 2017 sobre videolaringoscopía versus la ringoscopia directa para la intubación traqueal en niños, manifiesta que la videolaringoscopía no redujo el número de intentos de intubación en comparación con laringoscopía directa (DM -0.05; IC del 95\%: - 0.18 a 0.07; dos estudios; 427 intubaciones), concordando con nuestro estudio, en donde se evidenció que un 2.5\% de intubaciones con laringoscopia directa necesitaron dos o más intentos, versus un $11.6 \%$ con videolaringoscopía $(P=0.003)$ dato con poder estadístico. Dentro de este mismo estudio, sé reportó que la videolaringoscopía aumentó el éxito de la intubación en el primer intento en comparación con la laringoscopia directa, lo que difiere de nuestro estudio, en donde el $88.4 \%$ de intubaciones con $\mathrm{VL}$ se realizó en un intento, frente al $97.4 \%$ con $L D^{12}$.

Las complicaciones en la intubación según cada instrumento, no representaron diferencias considerables, mediante VL el $93.4 \%$ de intubaciones se realizó sin complicaciones versus un $97.4 \%$ de LD $(P=0.103)$. Esto interpreta que a pesar de la diferencia en las técnicas de laringoscopía, el Vividtrac y la mayoría de métodos de vídeo, guardan cierta semejanza estructural con el laringoscopio, lo que crea mayor familiaridad en el anestesiólogo para su uso evitando posibles lesiones en los pacientes y disminuyendo las complicaciones que podrían aparecer por nuevos dispositivos de video con técnicas distintas como los fibrobroncoscopios; sin embargo no olvidar lo anteriormente expuesto, donde se hacía referencia que una técnica adecuada de LD no garantiza el éxito en $V L^{13}$. Lingappan y cols, manifiesta que no hubo diferencias en la incidencia de traumatismo o complicaciones en las vías respiratorias debido a los intentos de intubación ( $p=0,10$ un estudio; 213 intubaciones), coincidiendo con nuestro estudio en donde no hubo diferencia estadísticamente significativa $(P=0.103)^{12}$.

Según el tiempo de intubación, observamos que, con LD el $94.2 \%$ se realizó en un tiempo menor a los 10 segundos, comparado con el $75.2 \%$ de la VL con $P<0.0001$. Un factor importante para esta estadística, fue la dificultad en la técnica de intubación con VL, el presente estudio reflejó un porcentaje de complejidad del $17.4 \%$ vs $1.9 \%$ de LD. La diferencia de los resultados en comparación con el artículo presentado, podrían representar un sesgo en el estudio, recordando que la presente investigación se llevó a cabo tanto con anestesiólogos experimentados como con médicos posgradistas en formación, lo que implica diferentes destrezas en cuanto a las técnicas de intubación. Los resultados demuestran la utilidad de la videolaringoscopía como una herramienta de enseñanza y aprendizaje ${ }^{14}$.

Un punto importante junto con la experiencia del operador, son los altos costos de comercialización de los equipos de video para particulares o establecimientos, lo que conduce al personal de salud a priorizar su experticia en el manejo del laringoscopio convencional por facilidad de acceso. Si los video laringoscopios estuvieran disponibles en todos los quirófanos y los costos de comercialización no supusieran una limitación, todos los anestesiólogos los usarian de rutina, según la sensación que deja el presente estudio ${ }^{15}$. Lingappan y cols, en referencia al tiempo de intubación manifestaron tiempos similares entre la video laringoscopia y la laringoscopia directa (diferencia de medias (DM) -0.62, intervalo de confianza (IC) del 95\%: 6.50 a 5.26), en discrepancia a nuestro estudio en donde los menores tiempos se observaron con laringoscopia directa, en parte por las razones antes mencionadas. 
Los resultados demuestran la predilección por LD para abordar una vía aérea pediátrica sin predictores de dificultad con un $56,2 \%$, resultado que es relativamente bajo para la tendencia de laringoscopía observada en salas de quirófano y en la literatura. Un factor considerable fue el compromiso de los anestesiólogos de participar en este estudio y ganar experiencia en métodos de video, lo que inclinaba su decisión al uso de VL, esto representó otra limitación en el estudio con una estadística que pudo ser considerablemente mayor para LD. Se necesitan estudios bien diseñados y con poder estadístico adecuado, para conocer el real beneficio y considerar la seguridad en el uso de video laringoscopios para el manejo de la vía aérea pediátrica de rutina, además de incluir en los estudios más instrumentos de video que brinden resultados más aproximados a la realidad, en donde el anestesiólogo tenga más opciones de elección según estas se adapten a sus habilidades y destrezas. Un dato adicional detectado y destacable en este estudio, fue la presencia de un caso de VAD de los 276 estudiados, lo que representa un porcentaje de $0.36 \%$; coincidiendo con Pérez Lara y cols, en su artículo de revisión, Vía aérea difícil en pediatría, en la cual manifiesta una incidencia del $0.3 \%$ para VAD en la población infantil2. Esta recolección de datos, al necesitar de la tecnología (Vividtrac-pantalla de video), representó una limitación en el estudio, al ser un instrumento de intubación con una vida de uso relativamente corta, ameritaba adquirir nuevas unidades del mismo en periodos cortos de tiempo, sin embargo, este tema no tuvo mayor connotación al momento de la toma de información. A pesar de las limitaciones del trabajo referidas, se considera un tamaño muestral importante para describir que las técnicas de intubación abracan dos puntos importantes. En primer lugar, la destreza y la experiencia del anestesiólogo en el procedimiento son factores decisivos en el resultado, la curva de aprendizaje con métodos de visualización indirecta implica mayores retos. En segundo lugar, la tecnología cambia constantemente el mundo en el que vivimos, y la medicina no está al margen de estos progresos, relacionarse con estos nuevos avances es abrir un abanico de opciones en donde el paciente y el especialista obtendrán un beneficio significativo. Finalmente, la utilización de VL para el manejo de una
VA de rutina esta desestimada, y se seguirá manteniendo la LD como la técnica predilecta. Esto significa que, si se utiliza el laringoscopio convencional para abordar una vía aérea, resulta indispensable tener cerca herramientas de apoyo adicional como los métodos de video, en caso de complicaciones no predecibles. Información que amerita sin dudas estar presente en futuros protocolos de manejo de VA sin predictores de dificultad. Desde el inicio de la formación como médicos hasta la especialización, algunos conceptos perduran a través del tiempo convirtiéndose en verdades intransigentes, como es el caso del laringoscopio, que desde el año 1941 en el que empezó su apogeo en el campo de la anestesia, se ha mantenido como el instrumento tradicional para abordar una VA, hasta los últimos años en los que el avance de la tecnología y los métodos de video, advierten variaciones importantes en la intubación orotraqueal, en la que además de priorizar el confort del especialista, será importante tener presente las repercusiones que pudieran presentarse a corto o largo plazo en los pacientes ${ }^{16,17}$.

\section{CONCLUSIONES}

- Se estudiaron pacientes de 5 a 17 años de edad, promedio estudio es de 9.83 años. El grupo de edad más frecuente fue de 5 a 10 años con el 61.2\%. Predominio del sexo masculino 58.3\%. El estado nutricional normal es más frecuente con el $56.9 \%$.

- La videolaringoscopía brinda una mejor visualización de la glotis y mejora en las condiciones de intubación, si bien en el presente estudio no aumentó el éxito de la intubación en primer intento, ni disminuyó el número de intentos necesarios, su utilidad por sobre la laringoscopia directa amerita estudios experimentales más extensos.

- Las complicaciones se presentan en porcentajes similares en las dos técnicas, teniendo en cuenta que la técnica para videolaringoscopia amerita una curva de aprendizaje más amplia.

- La videolaringoscopía no disminuyó los tiempos hasta una intubación satisfactoria, dependiente de muchos factores.

- El laringoscopio sigue siendo el gold standard en el manejo de la VA rutinaria, y los video laringoscopios limitan su uso a los casos de VAD. 


\section{INFORMACIÓN ADMINISTRATIVA DEL ARTÍ́CULO}

\author{
Abreviaturas \\ LD: Laringoscopia Directa \\ VA: Vía Aérea. \\ VAD: Vía Aérea Difícil. \\ VL: Video Laringoscopia
}

\section{Nota del Editor}

La Revista Ecuatoriana de Pediatría permanece neutral con respecto a los reclamos jurisdiccionales en mapas publicados y afiliaciones institucionales.

\section{Originalidad del artículo}

La Revista Ecuatoriana de Pediatría garantiza que el artículo es original y sin redundancia, el sistema antiplagio de nuestra revista reportó similitud menor al $4 \%$. El artículo proviene de una tesis de Especialidad cuya disertación completa está disponible en el repositorio de la Universidad de Cuenca: dspace.ucuenca/34562

\section{Acceso abierto}

Este artículo tiene la licencia de Creative Commons Attribution 4.0 CC-BY-NCSA., que permite el uso, el intercambio, la adaptación, la distribución y la reproducción en cualquier medio o formato, siempre que otorgue el crédito adecuado al autor original y a la fuente. Usted no puede hacer uso del material con propósitos comerciales. Se debe proporcionar un enlace a la licencia Creative Commons e indicar si se realizaron cambios. Las imágenes u otro material de terceros en este artículo están incluidos en la licencia Creative Commons del artículo. Para ver una copia de esta licencia, visite https://creativecommons.org/licenses/by-nc-sa/4.0/deed.es.

\section{DECLARACIONES ÉTICAS}

\section{Protección de personas:}

Los autores declaran que los procedimientos seguidos se conformaron a las normas éticas del comité de experimentación humana responsable y de acuerdo con la Asociación Médica Mundial y la Declaración de Helsinki.

\section{Confidencialidad de los datos:}

Los autores declaran que han seguido los protocolos de su centro de trabajo sobre la publicación de datos de pacientes.

\section{Consentimiento de publicación:}

Los autores han obtenido el consentimiento informado de los tutores de los pacientes y el respectivo asentimiento. Este documento obra en poder del autor de correspondencia. Se ha firmado la autorización para publicación del presente caso por parte de los padres.

\section{Conflictos de intereses}

Los autores declaran no tener conflictos de intereses.

\section{Agradecimientos}

A los integrantes del servicio de Anestesia del Hospital Vicente Corral Moscoso de Cuenca y a los pacientes y sus padres que aceptaron la participación del estudio.

\section{Financiamiento}

Los autores realizaron el financiamiento de los gastos incurridos en la producción de este artículo.

\section{Contribuciones de los autores}

JPPA: Idea de investigación, recolección de datos, escritura del artículo, correcciones editoriales.

JPP: Idea de investigación, análisis crítico, dirección de la investigación.

Todos los autores leyeron y aprobaron la versión final del manuscrito.

\section{INFORMACIÓN DE LOS AUTORES}

Juan Patricio Palacios Alvear Médico por la Universidad Católica de Cuenca, Especialista en Anestesia por la Universidad de Cuenca. Correo: juanpacho8903@gmail.com

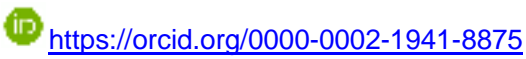

Juan Pablo Pacheco, Doctor en Medicina y Cirugía por la Universidad de Cuenca, Especialista en Anestesiología por la Universidad de Cuenca, Magíster en Investigación de la Salud, por la Universidad de Cuenca. 


\section{BIBLIOGRAFÍA}

1 Ríos Medina Á, Gómez LM, Aguirre Ospina O, Ocampo F. La vía aérea pediátrica: algunos conceptos para tener en cuenta en el manejo anestésico. Rev Colomb Anestesiol. 1 de julio de 201; 40(3):199- 202. SU: Scielo.co/4.n3

2. Pérez Lara J, Acosta Menoya A., Díaz Gener E., González Martínez Á. Vía aérea difícil en pediatría: signos predictores. Artículo de revisión. Revista de Ciencias Médicas de Pinar del Río, 2012;11(1):7282. SU: scielo.cu/S1561

3. Nørskov AK, Rosenstock CV, Wetterslev J, Astrup G, Afshari A, Lundstrøm LH. Diagnostic accuracy of anaesthesiologists' prediction of difficult airway management in daily clinical practice: a cohort study of 188064 patients registered in the Danish Anaesthesia Database. Anaesthesia. 2015;70(3):272-81 DOI: 10.1171/anae.12955. Epub 2014 Dec 16. PMID: 25511370.

4. Chaparro-Mendoza K, Luna-Montúfar CA, Gómez JM. Videolaringoscopios: ¿la solución para el manejo de la vía aérea difícil o una estrategia más? Revisión no sistemática. Revista Colombiana de Anestesiología. 1 de julio de 2015;43(3):225-33. SU: redalyc/ 195140440009

5. Ramírez-Acosta J, Torrico-Lara G, Encinas-Pórcel C. Índices predictores de vía aérea en pacientes obesos. Rev. Mex. Anestesiologia 2013;36(3):193- 201 SU: medigraphic/ 133

6. Salinas Rojas J, Granados Barrientos J, Valderrábano López A, Kume M, Ramírez Gorostiza A. Uso de videolaringoscopio Glidescope para manejo de vía aérea difícil en paciente con tumor de cuerdas vocales. Acta médica Grupo Ángeles 2017;15(3):234-238.

7. Alarcón-Almanza JM, Cano-Gálvez MA. Asociación de los grados de movilidad de la articulación atlanto-occipital con la clasificación de Cormack-Lehane como predictores de una vía aérea pediátrica difícil. Rev Mex Anest. 2016;39(1):15- 1 SU: medigraphic/ 63739

8. Echeverry Marín P, Engelhardt T. Algoritmo para el manejo de la vía aérea difícil en pediatría. Revista Colombiana de Anestesiología, 2014;42(4):325-334. ISSN: 0120-3347. SU: redalyc/ 195132030013

9. Healy DW, Maties O, Hovord D, Kheterpal S. A systematic review of the role of videolaryngoscopy in successful orotracheal intubation. BMC Anesthesiol. 2012;12:32. DOI: 10.1186/1471 2253- D-32. PMID: 23241277; PMCID: PMC3562270.
10. Law JA, Broemling N, Cooper RM, Drolet P, Duggan LV, Griesdale $D E$, et al. Canadian Airway Focus Group. The difficult airway with recommendations for management-part 1-difficult tracheal intubation encountered in an unconscious/induced patient. Can J Anaesth. 2013;60(11):1089-118. DOI: 10.1007/ s12630-013-0019-3. PMID: 24132407 Epub 2013 Oct 17. (PubMed).

11 Lema FE, Medina H, González C, Hoyos CE, Tafur BLA. Guía para la intubación con fibrobroncoscopio en un Hospital Universitario. Revista Colombiana de Anestesiología 2012;40(1):60-66. SU: elsevier/S0120334712700114

D. Lingappan K, Arnold JL, Fernandes CJ, Pammi M. Videolaryngoscopy versus direct laryngoscopy for tracheal intubation in neonates. Cochrane Database Syst Rev. 2018 Jun 4;6(6):CD009975. DOI: 10.1002/ 14651858.CD009975.pub3. PMID: 29862490; PMCID: PMC6513507.

13. Ramírez-Hernández ME, Hernández-Gutiérrez D, SánchezHernández E, et al. Videolaringoscopio Airtraq versus fibroscopio Bonfils para intubación orotraqueal electiva en paciente pediátrico con vía aérea normal. Rev Mex Anest. 2018;41(1):24-31 SU: medigraphic/ 77272

14. Healy DW, Picton P, Morris M, Turner C. Comparison of the glidescope, CMAC, storz DCI with the Macintosh laryngoscope during simulated difficult laryngoscopy: a manikin study. BMC Anesthesiol. 2012 Jun 21;12:11 DOI: 10.1186/1471-2253-12-11 PMID: 22720884; PMCID: PMC3519500.

15. Velázquez-Murillo G. Videolaringoscopio Artesanal Macintosh. Anestesia en México 2016;28(1):40-45. SU: scielo.mx/8772016000100040

16. Burkle CM, Zepeda FA, Bacon DR, Rose SH. A historical perspective on use of the laryngoscope as a tool in anesthesiology. Anesthesiology. 2004;100(4):1003-6. DOI: 10.1097/00000542200404000-00034. PMID: 15087639.

17. Burjek NE, Nishisaki A, Fiadjoe JE, Adams HD, Peeples KN, Raman VT, et al. PeDI Collaborative Investigators. Videolaryngoscopy versus Fiber-optic Intubation through a Supraglottic Airway in Children with a Difficult Airway: An Analysis from the Multicenter Pediatric Difficult Intubation Registry. Anesthesiology. 2017;127(3):432-440. DOI: 10.1097/ ALN.0000000000001758. PMID: 28650415. 